\title{
1 Antimicrobial use practices and opinions 2 of beef farmers in England and Wales
}

3 Charlotte Doidge ${ }^{1 *}$, Chris Hudson ${ }^{1}$, Rob Burgess ${ }^{2}$, Fiona Lovatt ${ }^{1}$, Jasmeet Kaler ${ }^{1}$

$4{ }^{1}$ School of Veterinary Medicine and Science, University of Nottingham, Leicestershire, 5 England

$6 \quad$ Evidence Group Ltd, Cumbria, England

*charlotte.doidge@nottingham.ac.uk

9

\section{Abstract}

\section{Background}

Limited research exists on antimicrobial use practices of beef farmers. This study aimed to investigate antimicrobial practices and perceptions of beef farmers in England and Wales, and identify drivers for higher antimicrobial use for the treatment of bovine pneumonia.

\section{Methods}

A survey was sent out in 2017 to beef farmers in England and Wales who supply to two abattoirs. Descriptive statistics were used to summarise the data. A logistic regression model was built to determine factors associated with treating $>5 \%$ of the predominant group in the herd with antimicrobials for pneumonia.

\section{Results}

There were a total of 171 useable responses. Most farmers reported using antimicrobials in $<5 \%$ of their herd for the treatment of common diseases. Most farmers (90\%) reported that they understood what antimicrobial resistance means, but only $55 \%$ were aware of critically important antimicrobials and $8 \%$ could name at least one critically important antimicrobial. Having a calf rearing enterprise and not considering Johne's disease when buying in cattle were associated with using antimicrobials to treat pneumonia in $>5 \%$ of the predominant group in the herd.

\section{Conclusion}

Self-reported antimicrobial use appears to be low in beef farms. However, some gaps in understanding aspects of antimicrobial stewardship by farmers were identified.

\section{Introduction}

Antimicrobial resistance (AMR) is a major threat to public health. The emergence of antimicrobial resistant bacteria coupled with the lack of development of new antimicrobials means that the effectiveness of current antimicrobials needs to be preserved. As a result, there have been many recommendations towards the responsible use of antimicrobials, both in human and veterinary medicine.[1] Examples in veterinary medicine include limiting the use of antimicrobials prophylactically, restricting the use of high priority critically important antimicrobials (CIAs) and the use of surveillance systems to monitor antimicrobial use (AMU).[2, 3] Antimicrobials include agents that act against bacteria, viruses, protozoa, parasites and fungi. In this paper, antimicrobial is used throughout but specifically refers to antibacterial antimicrobials. 
Despite the growing pressure of ensuring prudent use of antimicrobials, there are few data on how antimicrobials are used in the UK beef sector.[2] Reasons for this lack of data include the difficulty to distinguish between dairy and beef herds using sales data, the sheep and beef industries being highly interlinked, and the large variation between types of beef enterprises. Furthermore, veterinary prescription data may not correspond to what is actually used on the farm as farmers often keep stocks of antimicrobials in order to identify and treat disease themselves without veterinary supervision. The limited data available from the UK Veterinary Antimicrobial Resistance and Sales Surveillance (UK-VARSS) report suggests that beef herds may be higher users of antimicrobials than dairy herds. The report uses data collected from a convenience sample of 3,458 beef farms. However, the UK-VARSS report does not state how AMU was collated or how AMU was distinguished between dairy and beef cattle, or sheep and beef cattle. Therefore, it is unknown how reliable the data are. Whilst the quantification of antimicrobials used in the UK beef sector remains difficult, information on how beef farmers are using antimicrobials could be collected. For example, Brunton, et al. [4] reported the most frequently used types of antimicrobials and prophylactic treatment practices by UK dairy farmers. This type of information is not yet available for UK beef farmers.

To ensure responsible AMU in the beef sector, further understanding on why farmers use antimicrobials is needed. Whilst the opinions of UK dairy and pig farmers on various aspects of AMU have been studied, $[5,6]$ to the authors' knowledge there are no studies of UK beef farmers. Beef farms are typically more extensive than dairy or pig farms and therefore the perceptions and opinions on AMU and AMR of beef farmers may differ from what has previously been reported with pig and dairy farmers.

The aim of this study was to investigate the AMU knowledge, practices and opinions of beef producers in England and Wales, and identify drivers for higher AMU for the treatment of pneumonia.

\section{Methods}

\section{Survey design}

The survey was designed by $\mathrm{JK}, \mathrm{CH}$ and RB. Both an online and paper-based version of the survey were created. The online survey was produced using the Smartsurvey ${ }^{T M}$ platform and the paper version was produced using Microsoft Word. The survey was pilot tested on ten beef farmers and from their feedback, changes were made to some questions to improve clarity. The pilot surveys were not included in the final dataset. It was estimated that the survey would take around 20-25 minutes to complete. The survey was open from November 2017 to April 2018 and respondents were asked about their practices over the past twelve months.

Farmers were informed that the anonymised data generated from this survey were to be used and published for research purposes. Participation was voluntary and informed consent was gathered at the beginning of the survey by farmers agreeing to continue with the survey. All respondents were asked to answer sections on farm demographics, cattle health, AMU practices, and opinions on AMU and resistance. However, as some questions were not relevant to some enterprise types, respondents were not forced to answer every question. Hence, there were different response numbers for questions. There were 85 questions in total. The majority of questions were either nominal or ordinal with thirteen open-ended questions. An outline of the questionnaire sections relevant to this study is provided below. 
Farm demographics

This section included general questions such as geographic location of farm, other enterprises on the farm and type of production system.

Cattle health

In this section respondents were requested to rate common health problems on a 1-5 scale, where one was a significant health problem and five was not a health issue at all. A not applicable option was available.

\section{Antimicrobial use questions}

Sections were included for respondents to describe their AMU for pneumonia, lameness, scour, joint ill and mastitis over the last twelve months. A free text response was required for the most common antimicrobial product used for each disease. Respondents were also asked the most common group of cattle treated with antimicrobials, the proportion of cattle in this group treated with antimicrobials, and how they used antimicrobials. Respondents could select from prevention of disease (prophylaxis), as a group treatment in an outbreak situation (metaphylaxis) or as individual treatments.

Opinions on antimicrobial use

The section consisted of a series of statements related to AMU or resistance based on previous research on dairy farmers' opinions. $[6,7]$ The respondent's level of agreement with the statements was measured on a 5-point Likert scale from "Strongly Disagree" to "Strongly Agree". A don't know response was available.

Respondents were also asked how their AMU has changed, and how they expect their AMU to change in the next three years and to compare their AMU to other similar enterprises. They were also asked about their awareness of CIAs, and sources of information about AMR.

\section{Survey distribution}

The population of interest were all beef farmers in England and Wales. The population under study were farmers in England and Wales who supplied beef cattle to a British retailer through two abattoirs. Therefore, the inclusion criteria for this survey was beef producers whose contact details were available to two abattoirs that supply beef to one British retailer. Four hundred farmers were approached by one abattoir and 150 farmers were approached by the other abattoir. This represents $1.6 \%$ of the beef farms in England and Wales although not all the farmers we approached completed the survey.[8] The British retailer distributed the survey to farmers via a link to the online survey through email. Farmers who said that they did not have good internet access through phone communication with abattoir staff were sent a paper copy of the survey through the abattoir processors. Some responses were collected by abattoir staff by asking farmers to complete the survey when they brought their cattle in to the abattoir. Reminders to non-responders were sent by email via the abattoirs.

The study was approved by the University of Nottingham School of Veterinary Medicine and Science Ethics Committee (no 1850 160916).

\section{Data analysis}

Data cleaning, descriptive statistics and logistic regression were carried out in Stata 15.1 (Stata SE/15.1, Stata Corp., College Station, TX, USA). If there were duplicate entries from the same farm, the most complete response was kept for analysis. The responses to the open-ended questions in the cattle health section "Which antimicrobial product do you most commonly use for ...?" were categorised into the antimicrobial classes. Any answers which were not antimicrobials were removed from analysis. For descriptive 
analysis of numeric variables, the median and interquartile ranges were calculated and for categorical variables, contingency tables were produced.

\section{Multivariable logistic regression}

The dependent variable of interest was the proportion of the most commonly treated group in the herd treated with antimicrobials for pneumonia in the past twelve months. Respondents could select the breeding herd, pre-weaned calves, store cattle less than one year old or store cattle more than one year old as the group of animals they most commonly treat for pneumonia. Then respondents were asked what proportion of this group were treated for pneumonia. Respondents could select $<5 \%, 5-15 \%, 15-50 \%$ or $>50 \%$. The majority of respondents $(71 \%, 99 / 139)$ selected $<5 \%$. For modelling purposes a binary variable that was dichotomised at $5 \%$ of the most commonly treated group in the herd treated with antimicrobials for pneumonia was created.

Initially, a univariable analysis was carried out to explore factors most likely to be associated with antimicrobial use (Table 1 supplementary material). Variables with $\mathrm{p} \leq 0.1$ and more than 120 responses were considered for multivariable analysis, as well as potential confounders. A forward selection stepwise model building approach was used. Potential confounding variables were assessed through multiple regression analysis by adding and removing variables and evaluating changes to the regression coefficients. Only variables with $p \leq 0.05$ were selected to remain in the model.[9] Potential biologically relevant interaction terms were investigated by adding them into the model. The multivariable logistic regression model took the form of:

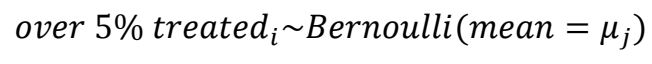

$$
\ln \left(\frac{\mu_{i}}{1-\mu_{i}}\right)=\alpha+\boldsymbol{\beta}_{\boldsymbol{i}} \boldsymbol{x}_{\boldsymbol{i}}
$$

Where over $5 \%$ treated $_{i}$ is whether the ith farmer treated over $5 \%$ of the most common group in the herd with antimicrobials for pneumonia, $\mu_{i}$ is the fitted probability of the outcome, $a$ is the intercept, and $\boldsymbol{\beta}_{\boldsymbol{i}}$ is the vector of coefficients corresponding to the vector of predictor variables (calf rearing enterprise, most common group treated for pneumonia, comparison of AMU to others, consideration of Johne's disease, digital cattle movements and pneumonia health challenge rating), $\boldsymbol{x}_{\boldsymbol{i}}$.

The Hosmer-Lemeshow test was carried out to test model fit. The variance inflation factor (VIF) and the tolerance was inspected for collinearity between variables.

\section{Results}

\section{General farm characteristics}

There were a total of 171 respondents, giving a response rate of $31 \%$. All of the respondents did not answer every question in the survey as some questions were not relevant to certain enterprise types. Of the 171 respondents, 72 had a suckler herd, 42 had a calf rearing herd and 124 had a growing and finishing herd as part of their enterprise. Almost half of farmers were aged between 46-65 (48\%, 82/171), 30\% (50/171) were aged between 26-45, 17\% (29/171) were over 65 and 5\% (10/171) were under 25 years old. Additionally almost half of farms were based in the West Midlands (48\%, 81/171), 22\% (37/171) were in Northern England, 19\% (33/171) were in Wales, and $11 \%(19 / 171)$ were in the South or Eastern England. The majority of farms also comprised of a sheep enterprise $(64 \%, 110 / 171)$. 


\section{Suckler herds}

Of the 72 enterprises with suckler herds, the median number of suckler cows in the herd was $65(\mathrm{IQR}=32,90)$. The median suckler target finishing ages was 20 months $(\mathrm{IQR}=18,24)$.

\section{Calf rearing herds}

The median number of dairy calves bought annually was 85 (IQR=50, 170). The median dairy target finishing age was 22 months $(\mathrm{IQR}=19,25)$.

\section{Finisher herds}

The median annual number of weaned calves (less than 1 year old) bought was 60 $(\mathrm{IQR}=25,100)$. The median number of store cattle (greater than 1 year old) bought was $150(\mathrm{IQR}=55,430)$. The target finished age for finisher cattle was 24 months (IQR=21, 27.5).

\section{Cattle health}

The main source of information and advice on the health of cattle for $74 \%$ of farmers was their local veterinarian (119/159). Table 1 shows how respondents rated health issues in terms of challenges to their herd, where 1 is significant health issue and 5 is not a health issue at all.

Table 1: Cattle farmers' ratings for health issues in terms of challenges to their herd ( $1=$ Significant health issue, $5=$ Not a health issue at all)

\begin{tabular}{|c|c|c|c|c|c|c|}
\hline $\begin{array}{l}\text { Health } \\
\text { problem }\end{array}$ & $\mathbf{N}^{\mathbf{a}}$ & 1 & 2 & 3 & 4 & 5 \\
\hline Pneumonia & 170 & $25.90 \%$ & $14.10 \%$ & $27.10 \%$ & $18.20 \%$ & $14.70 \%$ \\
\hline Liver fluke & 164 & $15.20 \%$ & $15.20 \%$ & $22.00 \%$ & $24.40 \%$ & $23.20 \%$ \\
\hline Worms & 162 & $11.70 \%$ & $14.20 \%$ & $31.50 \%$ & $22.20 \%$ & $20.40 \%$ \\
\hline Fertility & 79 & $10.10 \%$ & $5.10 \%$ & $17.70 \%$ & $34.20 \%$ & $32.90 \%$ \\
\hline Coccidiosis & 118 & $8.50 \%$ & $6.80 \%$ & $15.30 \%$ & $24.60 \%$ & $44.90 \%$ \\
\hline Lameness & 167 & $8.40 \%$ & $12.00 \%$ & $22.20 \%$ & $38.90 \%$ & $18.60 \%$ \\
\hline $\begin{array}{l}\text { Navel/joint } \\
\text { ill }\end{array}$ & 111 & $7.20 \%$ & $7.20 \%$ & $9.00 \%$ & $21.60 \%$ & $55.00 \%$ \\
\hline Mastitis & 87 & $6.90 \%$ & $6.90 \%$ & $17.20 \%$ & $36.80 \%$ & $32.20 \%$ \\
\hline $\begin{array}{l}\text { Diarrhoea } \\
\text { calves } \\
\text { under } 1 \\
\text { month }\end{array}$ & 104 & $6.70 \%$ & $13.50 \%$ & $16.30 \%$ & $34.60 \%$ & $28.80 \%$ \\
\hline
\end{tabular}

\section{a Number of responses differs as some health problems were not applicable to all enterprise types}

\section{Antimicrobial management of pneumonia}

Only $1 \%(2 / 145)$ reported using antimicrobials as a preventative measure for pneumonia (prophylaxis), and 1\% used antimicrobials as a group treatment following an outbreak of pneumonia (metaphylaxis). The most common group of cattle treated with antimicrobials was relatively evenly distributed between store cattle less than 1 year old ( $37 \%$ $52 / 139)$, pre-weaned calves (37\% 51/139) and store cattle over 1 year old ( $26 \%$ $36 / 139)$. Twenty-eight percent of farms reported treating over $5 \%$ of the group that they most commonly treat with antimicrobials for pneumonia (40/139). The antimicrobial classes that were most commonly named by farmers for the treatment of pneumonia are presented in Figure 1. There were eleven farmers who named either vaccines or anti- 
210 inflammatories instead of an antimicrobial and therefore their answers were excluded

211 from the analysis of this section.

\section{Figure 1: Antimicrobial classes most commonly used by farmers for the 213 treatment of pneumonia $(\mathbf{N}=132)$}

\section{Antimicrobial management of diarrhoea}

215 This health issue was relevant to 64 farms who responded to the survey. Antimicrobials were reportedly used to treat sick individual animals on $95 \%(61 / 64)$ of farms. Sixteen percent $(8 / 63)$ of farms reported treating over $5 \%$ of their most commonly treated group with antimicrobials for calf diarrhoea. Penicillins were the most common antimicrobial class used to treat calf diarrhoea $(65 \%, 36 / 55)$. There were sixteen farmers who named treatments which did not contain antimicrobials and therefore were excluded from descriptive analysis of this section.

\section{Antimicrobial management of other diseases}

Five percent (3/57) and $4 \%(2 / 55)$ of farmers reported treating over $5 \%$ of their breeding herd with antimicrobials for calving related disease and mastitis, respectively. Seven percent $(5 / 72)$ and $8 \%(11 / 142)$ of farmers reported treating over $5 \%$ of cattle in the predominant group in the herd with antimicrobials for joint ill and lameness, respectively. None of the respondents reported the use of CIAs for treatment of calving related disease, mastitis, lameness or joint ill.

Antimicrobial knowledge, opinions and perceptions around AMU and AMR

Over half of farmers were aware of CIAs (55\% 93/169). Only 9\% (15/169) of farmers were able to name at least one CIA listed by the European Medicine Agency. A small number of farmers thought that their antimicrobial usage had increased in the past three years (4\% 6/168), 37\% (62/168) thought that their antimicrobial usage had remained roughly the same, and $59 \%(100 / 168)$ thought that their antimicrobial usage had reduced. The majority of respondents expect that their AMU will remain roughly the same in the next three years $(62 \% 104 / 168)$; whilst $37 \%(63 / 168)$ of respondents expect that their AMU will reduce.

The main source where farmers had heard of AMR in the past twelve months was print or other media $(74 \%, 126 / 171)$. Under half of respondents had heard about AMR from their veterinarian $(44 \%, 75 / 171)$.

Just over half of farmers thought they had the support they needed to reduce AMU in their beef enterprise (52\% 86/166), 31\% were not sure and $17 \%$ thought they did not have the support they need. When asked what additional support would help to reduce AMU in their beef herd, $42 \%$ would have liked more information on disease control $(71 / 171), 41 \%$ would have liked more information on different types of antimicrobial (70/171), 29\% would have liked one-to-one advice on reducing disease in their herd (49/171), 25\% would have liked clearer messages about goals on AMU (43/171) and $20 \%$ would have liked benchmarking data on AMU in beef enterprises (35/171).

Just under $40 \%(66 / 166)$ of farmers agreed that antimicrobials were beneficial to prevent diseases in their herd. Almost a quarter of farmers agreed that it is acceptable to use antimicrobials to prevent disease in animals (24\% 40/166). Almost ninety percent $(149 / 166)$ of farmers believed that they understood what AMR means. Table 2 presents the ratings of each antimicrobial statement. 

use and antimicrobial resistance

Statement
$\mathbf{N}$

$\%$

Strongly agree or agree

\begin{tabular}{lll}
$\begin{array}{l}\text { \% } \\
\text { Neutral }\end{array}$ & $\begin{array}{l}\% \\
\text { Strongly } \\
\text { disagree } \\
\text { or } \\
\text { disagree }\end{array}$ & $\begin{array}{l}\text { \%o } \\
\text { Don't } \\
\text { know }\end{array}$ \\
\hline 15.6 & 40.4 & 4.0
\end{tabular}

$\% \quad \%$

disagree

disagree

Use of antimicrobials is
beneficial to prevent disease
in my herd

Use of antimicrobials is

166

34.9

15.7

45.8

3.6

beneficial to maximise

productivity of my herd

Use of antimicrobials is

166

66.3

20.5

10.8

2.4

beneficial to the welfare of

my herd

\begin{tabular}{|c|c|c|c|c|c|}
\hline $\begin{array}{l}\text { It is ok to use antimicrobials } \\
\text { to treat sick individual } \\
\text { animals }\end{array}$ & 166 & 93.4 & 0.0 & 4.2 & 2.4 \\
\hline $\begin{array}{l}\text { It is ok to use antimicrobials } \\
\text { to prevent disease in } \\
\text { animals }\end{array}$ & 166 & 24.1 & 16.9 & 53.6 & 5.4 \\
\hline $\begin{array}{l}\text { Society thinks farmers use } \\
\text { too much antimicrobials }\end{array}$ & 166 & 56.0 & 19.9 & 10.2 & 13.9 \\
\hline $\begin{array}{l}\text { Using less antimicrobials } \\
\text { makes me a good farmer }\end{array}$ & 166 & 41.6 & 27.1 & 19.3 & 12.1 \\
\hline $\begin{array}{l}\text { I understand what } \\
\text { antimicrobial resistance } \\
\text { means }\end{array}$ & 166 & 89.7 & 3.6 & 3.6 & 3.0 \\
\hline $\begin{array}{l}\text { Preventative use of } \\
\text { antimicrobials can } \\
\text { contribute to antimicrobial } \\
\text { resistance }\end{array}$ & 166 & 71.1 & 9.0 & 6.6 & 13.3 \\
\hline $\begin{array}{l}\text { Curative use of } \\
\text { antimicrobials can } \\
\text { contribute to antimicrobial } \\
\text { resistance }\end{array}$ & 166 & 34.9 & 22.3 & 26.5 & 16.3 \\
\hline $\begin{array}{l}\text { The use of antimicrobials in } \\
\text { animals can contribute to } \\
\text { antimicrobial resistance in } \\
\text { people }\end{array}$ & 166 & 48.8 & 19.3 & 10.2 & 21.7 \\
\hline $\begin{array}{l}\text { Reduction in the use of } \\
\text { antimicrobials could be } \\
\text { achieved with better } \\
\text { management or vaccines }\end{array}$ & 166 & 72.3 & 12.7 & 7.8 & 7.2 \\
\hline $\begin{array}{l}\text { If every beef farmer } \\
\text { followed best practice, there } \\
\text { would be less resistant } \\
\text { bacteria }\end{array}$ & 166 & 39.2 & 22.3 & 17.5 & 21.1 \\
\hline $\begin{array}{l}\text { I have the skills and } \\
\text { knowledge needed to reduce } \\
\text { antimicrobials in my herd }\end{array}$ & 166 & 41.8 & 25.9 & 10.2 & 12.1 \\
\hline $\begin{array}{l}\text { Reducing the use of } \\
\text { antimicrobials in my herd } \\
\text { over the next year would be } \\
\text { difficult }\end{array}$ & 166 & 43.4 & 27.1 & 21.8 & 7.8 \\
\hline $\begin{array}{l}\text { Reducing antimicrobial } \\
\text { usage in my herd would } \\
\text { have costs }\end{array}$ & 166 & 44.0 & 22.9 & 21.7 & 11.5 \\
\hline
\end{tabular}


261 A multivariable logistic regression model was built to estimate the associations of farmer practices and opinions on treating over $5 \%$ of the most common group in the herd with antimicrobials for pneumonia. The results are presented in Table 3.

264 Table 3: Results of multivariable logistic regression for treatment of over $\mathbf{5 \%}$ of 265 the herd with antimicrobials for pneumonia $(\mathbf{N}=129)$

\begin{tabular}{|c|c|c|c|}
\hline & $\mathrm{N}$ & Odds Ratio $(95 \% \mathrm{Cl})$ & $P>z$ \\
\hline \multicolumn{4}{|l|}{$\begin{array}{l}\text { Most common group treated } \\
\text { for pneumonia }\end{array}$} \\
\hline Not pre-weaned calves & 81 & Ref & \\
\hline Pre-weaned calves & 48 & $14.16(3.41,58.83)$ & $<0.001$ \\
\hline \multicolumn{4}{|l|}{ Enterprise type } \\
\hline Not calf rearing enterprise & 95 & Ref & \\
\hline Calf rearing enterprise & 34 & $5.20(1.41,19.14)$ & 0.013 \\
\hline $\begin{array}{l}\text { Compare AMU } \\
\text { AMU the same or higher } \\
\text { than similar enterprises } \\
\text { AMU less than other } \\
\text { enterprises }\end{array}$ & $\begin{array}{l}51 \\
78\end{array}$ & $\begin{array}{l}\text { Ref } \\
0.29(0.05,0.88)\end{array}$ & 0.041 \\
\hline $\begin{array}{l}\text { Consider Johne's disease } \\
\text { Sometimes or always } \\
\text { consider Johne's }\end{array}$ & 92 & Ref & \\
\hline Never consider Johne's & 37 & $5.09(1.31,19.14)$ & 0.019 \\
\hline \multicolumn{4}{|l|}{$\begin{array}{l}\text { Collect cattle movements } \\
\text { digitally }\end{array}$} \\
\hline Yes & 100 & Ref & \\
\hline No & 29 & $4.55(1.13,18.26)$ & 0.033 \\
\hline \multicolumn{4}{|l|}{ Pneumonia health challenge } \\
\hline Health problem (Score 1-2) & 56 & Ref & \\
\hline Not a health problem (Score & 73 & & \\
\hline $3-5)$ & & $0.27(0.09,0.83)$ & 0.023 \\
\hline Intercept & & $0.21(0.06,0.81)$ & 0.23 \\
\hline
\end{tabular}

When the age group most commonly treated with antimicrobials for pneumonia was preweaned calves, the odds of reportedly treating over $5 \%$ of the herd with antimicrobials were 14.16 times higher $(C I=3.41,58.83)$ compared to when other age groups were most commonly treated.

Farms where calf-rearing was part of the production system had 5.20 times higher odds of treating more than $5 \%$ of the group for pneumonia ( $C I=1.41,19.14)$ compared to respondents without a calf rearing enterprise.

274 For respondents not considering Johne's disease when buying in new cattle, the odds of reportedly treating over $5 \%$ of the herd with antimicrobials were 5.09 times higher

$276(\mathrm{CI}=1.31,19.14)$ compared to respondents who sometimes or always considered

277 Johne's disease when buying in new cattle. 
When pneumonia was not a health problem for the herd the odds of treating over $5 \%$ of the herd with antimicrobials was $73 \%$ lower $(C I=0.09,0.83)$.

280 The odds of treating over $5 \%$ of the herd with antimicrobials was $69 \%(C I=0.05,0.88)$ lower in farmers who thought they used less antimicrobials than other enterprises compared to farmers who thought they used a similar amount or more antimicrobial than other enterprises.

The odds of treating over $5 \%$ of the herd with antimicrobials were 4.55 times ( $C I=1.13$, 18.26) higher when farmers did not record cattle movements digitally, compared to those who did.

The Hosmer-Lemeshow test gave a p-value of 0.5 , indicating that the model fit the data well. The VIF and tolerance values of the variables used in the logistic regression indicated that there were no collinearity problems.

This study provides insight on AMU practices of beef farms in the UK. To the authors knowledge it is the first study in the UK to present the opinions of beef farmers towards AMU and resistance and to report drivers for increased AMU for the treatment of pneumonia. Most farmers reported that they treated less than $5 \%$ of the herd with antimicrobials for common health problems, suggesting that AMU was low. This is perhaps in contrast with the figures reported by RUMA, where beef farmers had a higher AMU than dairy farmers. [10] Reasons for this disagreement could be due to the difference in study designs or that farmers in this survey under reported AMU due to social desirability bias.[11]

One of the key findings in this study was that whilst few farmers reported using antimicrobials for prevention of disease, many farmers may think this is still appropriate practice. Around $24 \%$ of farmers thought that it was acceptable to use antimicrobials to prevent disease, and $40 \%$ thought that antimicrobials were beneficial to prevent diseases in their herd. The proportion of farmers who agreed with preventative antimicrobial use may be relatively high as in cases such as an outbreak of respiratory disease it may be prudent to treat a group of animals before clinical signs are apparent (metaphylaxis). As respondents were only asked about preventative AMU, the authors were unable to distinguish differing opinions on metaphylactic and prophylactic AMU. The difference between attitudes towards prophylactic or metaphylactic AMU and actually carrying out the practice may be because farmers do not want to use antimicrobials for reasons such as cost, time or that they do not think that the disease levels in their herd warrant such use. Farmers may think that antimicrobials would be beneficial for prevention of disease in their herd but do not undertake this practice as they are aware of the risks of AMR. Alternatively, farmers may not want to state that they use antimicrobials for prevention even when they do, as AMU in agriculture has had considerable attention over recent years.[1] A further reason for this difference is that there may be multiple people employed on a farm and the person filling in the survey may have not known about the AMU in separate management groups over the twelve month period.

Most AMU tended to be for curative reasons with antimicrobial classes that are low risk to public health such as penicillin and tetracyclines. The low use of third generation cephalosporins and fluoroquinolones may be why only $55 \%$ of farmers were aware of CIAs, and even fewer could name one. A slightly higher proportion (60\%) of UK pig farmers were aware of CIAs.[12] Although beef farmers seem to be low users of CIAs, it 
is still important to improve the awareness levels in case their veterinarian prescribes them CIAs in the future.

Most farmers (90\%) said that they understood what AMR means. A similar level of understanding was reported by UK dairy farmers. [13] However, levels of reported understanding around AMR may not be true as Higham [13] demonstrated that only $55 \%$ of dairy farmers could give an accurate description of AMR despite most of them saying they understand what AMR means. In order to investigate whether this is also true for beef farmers their knowledge and understanding of AMR requires further exploration.

It appears that many beef farmers have already taken steps to reduce their antimicrobial usage. Very few beef farms in this study were using antimicrobials for prevention of disease in their herd with 63\% reporting that they had reduced their AMU in the past three years. However, additional support for UK beef farmers may be needed if further reductions or refinements in AMU are required as a lower proportion (37\%) of farmers expect their AMU to reduce in the next three years. Indeed, only $52 \%$ of farmers thought they had the support they needed to further reduce their AMU. AMU is under the control of the veterinarian and antimicrobials on farm must be prescribed by the veterinarian who has the animals 'under their care' (RCVS legislation). In practice, the veterinarian does not attend every animal that requires antimicrobials but develops a relationship with the farmer and establish protocols that the farmer follows. This survey shows that under half the farmers had heard about AMR from their veterinarian. This may be because there is often a lack of contact between the veterinarian and beef farmer,[14] and previous work has identified that farmers may be unwilling to have regular veterinary visits to their farm. $[15,16]$ The most commonly selected area where farmers would like more support around AMU reduction was more information on disease control, suggesting that some farms are unable to reduce their AMU further without compromising animal welfare. The veterinarian is best placed to advice on reducing disease in their herd.[15, 16] Clearly, this strategy necessitates all veterinarians understanding good practice and delivering appropriate advice with a proactive relationship between the veterinarian and farmer. Although veterinarians have an essential role in ensuring good antimicrobial stewardship, barriers to a proactive relationship between veterinarians and farmers need to be tackled first.

Sixty-four farms were using antimicrobials for the treatment of calf diarrhoea. A further sixteen farmers indicated treatments other than antimicrobials when asked about the most common antimicrobial used to treat diarrhoea. These results illustrate two important findings. First, treatment of uncomplicated diarrhoea with antimicrobials is discouraged [17] though farmers in this study appear to be using them anyway. This may be because farmers only ask for their veterinarians' advice in complex cases and due to the lack of contact with the veterinarian in cases of uncomplicated diarrhoea, the farmer remains unaware that antimicrobial treatment is unnecessary. Second, some farmers were unable to distinguish between antimicrobials and other treatments such as endoparasiticides or anti-inflammatories. This has obvious important implications for potential inaccuracies in farmer-reported AMU.

To understand why some farms may have an increased need to use more antimicrobials than others, a logistic regression analysis was carried out to determine factors 
health issue reported in the survey and was a disease that covered the three enterprise types. Drivers for increased AMU for the treatment of pneumonia included having a calfrearing enterprise and pre-weaned calves being the most common group of cattle treated with antimicrobials. Type of production system was also identified as a driver for AMU in Tennessee cattle producers.[18] Having a calf-rearing enterprise may increase AMU as calves from a mix of farms are transported to a calf-rearing enterprise at a young age, which is a risk factor for development of bacterial pneumonia infection.[19] Indeed, a higher rating for pneumonia as a health challenge, which suggests a high prevalence of pneumonia within the herd, was also a driver for increased AMU.

It appears that some farmers are aware of how much antimicrobials they use compared to other farms, as those who thought their AMU was less than other similar enterprises were less likely to treat over $5 \%$ of the most commonly treated group with antimicrobials for pneumonia. Similarly, pig farmers who used more antimicrobials estimated their own usage as higher than other pig farmers.[20]

Some management practices were associated with AMU. The practice of never considering Johne's disease when buying in cattle significantly increases the likelihood of treating over $5 \%$ of the herd with antimicrobials for pneumonia. This may be because a relaxed attitude to biosecurity is associated with other management decisions that increase the risk of pneumonia in calves.

The other management factor associated with proportion of herd treated with antimicrobials for pneumonia was use of digital cattle movements. Cattle movements were the most common information reported by farmers digitally, possibly because in the UK the recording and reporting of all cattle moving on or off the farm is mandatory. Use of electronic identification has previously been associated with lower lameness levels in sheep. [21] The use of digital management tools may be associated with reduced disease levels within the herd and consequently in lower AMU.

\section{Study limitations}

The sample was biased geographically due to the location of the two abattoirs represented. The number of herds was small but herd size large.[8] Therefore, comparison of these results with those from other populations may not be appropriate. Despite this the associations reported in this study needs further investigation. The study results highlight the importance of farmer's beliefs regarding AMU and AMR and that these need to be understood and tackled before longer term changes can be seen in the industry.

There were no exclusion criteria for respondents in terms of the role they had on their farm. Therefore, some respondents may not necessarily be responsible for all the animal groups on their farm and not know all the antimicrobial treatments given on their farm. Respondents may have interpreted the proportion of animals treated within the last twelve months differently.

As the information in the survey was self-reported, there may be some social desirability bias, particularly with sensitive topics such as inappropriate AMU which may be perceived as a socially "undesirable" behaviour.[11] The survey was based on general health management rather than explicitly focusing on AMU, which should mean that the survey was not skewed towards farmers with a specific interest in AMU. Farmers may have difficulty recalling practices in the past twelve months so may be affected by recall bias. Questions were asked about management in the past twelve months and therefore date of questionnaire completion was included in the logistic regression analysis to check if this had a significant effect on the dependent variable. Date was not statistically significant $(p=0.93)$ so was not included in the final model. 
424 The results of this study suggest that AMU in beef farms is low and the majority of farmers are using antimicrobials for curative reasons rather than for prevention of disease. Farmers' reported understanding of AMR was high but awareness of CIAs was relatively low and could be improved. Drivers for increased AMU were identified in the study, which may help veterinarians and farmers better understand how to improve antimicrobial stewardship within the beef industry.

\title{
Acknowledgements
}

We would like to thank all farmers who completed the survey. We would also like to thank Dr Mandy Nevel for her useful comments on the manuscript.

\section{Funding}

\author{
CD is supported by a studentship from AHDB.
}

\section{Competing Interests}

The authors declare no competing interests

\section{References}

1. O'Neill J. Tackling drug-resistant infections globally: Final report and recommendations. 2016. Available from: https://amrreview.org/sites/default/files/160525 Final\%20paper with\%20cover.pdf. 2. $\quad$ RUMA. RUMA Targets Task Force Report. 2017.

3. Speksnijder DC, Mevius DJ, Bruschke CJM, et al. Reduction of Veterinary Antimicrobial Use in the Netherlands. The Dutch Success Model. Zoonoses Public Health. 2015;62(s1):79-87.

4. Brunton L, Duncan D, Coldham N, et al. A survey of antimicrobial usage on dairy farms and waste milk feeding practices in England and Wales. Vet Rec. 2012;171(12): 296 .

5. Coyne L, Pinchbeck $G$, Williams $N$, et al. Understanding antimicrobial use and prescribing behaviours by pig veterinary surgeons and farmers: a qualitative study. Vet Rec. 2014;175(23):593.

6. Jones $P$, Marier $E$, Tranter $R$, et al. Factors affecting dairy farmers' attitudes towards antimicrobial medicine usage in cattle in England and Wales. Prev Vet Med. 2015;121(1-2):30-40.

7. Swinkels J, Hilkens A, Zoche-Golob V, et al. Social influences on the duration of antibiotic treatment of clinical mastitis in dairy cows. J Dairy Sci. 2015;98(4):2369-80. 8. AHDB. UK Yearbook 2018 Cattle. 2018.

9. Vandenbroucke JP, Von Elm E, Altman DG, et al. Strengthening the Reporting of Observational Studies in Epidemiology (STROBE): explanation and elaboration. PLoS Med. 2007; 4(10):e297.

10. RUMA. Targets Task Force: One Year On. 2018.

11. De Vaus D. Surveys in social research. New York: Routledge; 2013.

12. Coyne LA, Latham SM, Dawson S, et al. Exploring Perspectives on Antimicrobial Use in Livestock: A Mixed-Methods Study of UK Pig Farmers. Frontiers in Veterinary Science. 2019;6(257).

13. Higham LE, Deakin A, Tivey E, et al. A survey of dairy cow farmers in the United Kingdom: knowledge, attitudes and practices surrounding antimicrobial use and resistance. Vet Rec. 2018;183(24):746-. 
14. Lowe P. Unlocking Potential: A Report on Veterinary Expertise in Food Animal 470 Production: to the Vets and Veterinary Services Steering Group: Vets and Veterinary 471 Services Steering Group; 2009.

472 15. Kaler J, Green LE. Sheep farmer opinions on the current and future role of 473 veterinarians in flock health management on sheep farms: A qualitative study. Prev Vet 474 Med. 2013;112(3):370-7.

475 16. Ruston A, Shortall O, Green M, et al. Challenges facing the farm animal veterinary 476 profession in England: A qualitative study of veterinarians' perceptions and responses.

477 Prev Vet Med. 2016;127:84-93.

478 17. Constable PD. Antimicrobial Use in the Treatment of Calf Diarrhea. $J$ Vet Intern 479 Med. 2004;18(1):8-17.

480 18. Ekakoro JE, Caldwell M, Strand EB, et al. Drivers, alternatives, knowledge, and 481 perceptions towards antimicrobial use among Tennessee beef cattle producers: a 482 qualitative study. BMC Vet Res. 2019;15(1):16.

483 19. Caswell JL. Failure of Respiratory Defenses in the Pathogenesis of Bacterial 484 Pneumonia of Cattle. Vet Pathol. 2014;51(2):393-409.

485 20. Visschers VHM, Postma M, Sjölund M, et al. Higher perceived risk of 486 antimicrobials is related to lower antimicrobial usage among pig farmers in four 487 European countries. Vet Rec. 2016;179(19):490.

488 21. Lima E, Hopkins T, Gurney E, et al. Drivers for precision livestock technology 489 adoption: A study of factors associated with adoption of electronic identification 490 technology by commercial sheep farmers in England and Wales. PLOS ONE.

$491 \quad 2018 ; 13(1): \mathrm{e} 0190489$. 